\title{
Marktforschung in der Praxis: Kundenpräferenzen beim Kauf eines neuen Fernsehgerätes
}

\author{
Sascha Bartels, Fabian Bräuner, Mario Glowik, Roman Hawlitzky, Claudia Kottke, \\ Claudia Liedtke, Julia Mücke, Matthias Schidzick, Christopher Weiß
}

\section{Zusammenfassung}

In der heutigen Zeit sind Medien aller Art im alltäglichen Leben anzutreffen. Ob früh am Morgen die frisch gedruckte Zeitung, das Radio in der Küche, der Lieblingsfilm im Fernsehen oder das Internet via Mobiltelefon. Viele Menschen können sich heutzutage ein Leben ohne Mobiltelefon oder Fernsehgerät nicht mehr vorstellen. Die Industrie hat dies erkannt und bringt fast täglich neue Weiterentwicklungen auf den Markt. Smartphones, die beinahe so viel können wie ein Laptop oder Fernseher, die den Zuschauer via 3D-Technologie in das Geschehen eintauchen lassen, entwickeln sich zu Standardprodukten.

Bei der heutigen Geschwindigkeit der Neuentwicklungen und den immer kürzer werdenden Produktlebenszyklen ist es besonders wichtig, den Endverbraucher vom Mehrwert zu überzeugen. In diesem Zusammenhang ist es notwendig herauszukristallisieren, worauf der Endkunde Wert legt und welche Informationsschwächen er hat. Hierzu haben acht Studierende der TH Wildau aus dem Fachbereich Betriebswirtschaft/Wirtschaftsinformatik eine empirische Studie entwickelt.

\section{Abstract}

In your everyday life, media of all kinds are found. Whether early in the morning, freshly printed newspaper, the radio in the kitchen, favorite movies on TV or the Internet via mobile phone. Many people can not imagine a life without mobile phone or TV. The industry has recognized that and almost every day they have new developments for the market. Smartphones have nearly as much as functions as a laptop or a television and are able to bring the viewers into actions via $3 \mathrm{D}$ technology as a standard.

Because of the speed of new developments and the shortening product life cycle it is especially important to convince the consumer «s added value. In this case it is important to have a look what is significant for the end consumer and what lack of information does the consumer have. Concerning these problems, eight students of the University of Applied Science Wildau from the Department of Business Administration and Business Informatics designed an empirical study.

\section{Zum Begriff der Marke}

Die hohe Relevanz von Marken ist für den Erfolg eines Unternehmens unbestreitbar (Esch et al. 2006: 2). Die vielleicht bedeutendste und nachhaltigste Marketingleistung besteht darin, eine stabile Marke einzuführen und diese für die Zukunft zu erhalten und zu sichern (Kotler et al. 2001: 736).

Es lassen sich, nach Meffert et al. (2002: 6) drei Begriffsverständnisse von Marken unterscheiden:

- die Marke als ein gewerbliches Schutzrecht,

- die Marke als markiertes Produkt,

- die Marke als produktübergreifendes Konzept.

Versteht man die Marke als ein produktübergreifendes Konzept, ergibt sich folgende Definition: Die Marke kann als ein, in der Psyche des Konsumenten und sons- tiger Bezugsgruppen der Marke fest verankertes, unverwechselbares Vorstellungsbild von einem Produkt oder einer Dienstleistung definiert werden (Meffert et al. 2002: 6). Der Markenaufbau muss als ein strategisches, langfristig ausgelegtes Konzept betrachtet werden. Dieser Prozess kann nur gelingen, wenn ein Markenbild bzw. Markenimage in den Köpfen der Menschen erzeugt wird. In diesem Zusammenhang spricht man von einer Markenidentität (Hohn 2008: 136).

Eine klare Identität und die sachlich-funktionale Kompetenz einer Marke schafft notwendiges Vertrauen beim Verbraucher. Eine Markenidentität kann aber nicht kurzfristig entwickelt werden. Der Entwicklungsprozess einer Marke ist langfristig auf mehrere Jahre oder sogar Jahrzehnte angelegt (Adjouri 2002: 125). Der richtige Aufbau einer Marke lohnt sich, denn gut 
geführte Marken »leben länger « als jede Produktionsanlage und jeder Produktlebenszyklus (Kotler et al. 2001: 741).

\section{Begriffsbestimmung und Anforderun- gen an eine moderne Marktforschung}

Auf Grund verkürzter Produkt- und Technologielebenszyklen steigt die Komplexität an eine zeitnahe, effiziente Marktforschung. Unterstellt man eine marktorientierte Unternehmenskultur, so ist es Hauptaufgabe der Marktforschung, dem Marketing-Management auf empirischem Wege die Informationsgrundlage für die absatzpolitische Ziel- und Maßnahmenplanung bereitzustellen. Unter Marktforschung versteht man das systematische Sammeln, Aufbereiten, Analysieren und Interpretieren von Daten über Märkte und Marktbeeinflussungsmöglichkeiten zum Zweck der Absicherung von Marketingentscheidungen. Außerdem ist die Marktforschung durch den systematischen Einsatz wissenschaftlicher Untersuchungsmethoden gekennzeichnet. Ziel ist nicht nur die sorgfältige Beschreibung von Märkten, sondern auch die Gewinnung von Aussagen über Ursache-Wirkungsbeziehungen, z. B.: »Wie wirkt der Einsatz von Marketing-Maßnahmen auf das Käuferverhalten?«(Böhler 2004: 19).

Man unterscheidet grundsätzlich zwischen quantitativer und qualitativer Marktforschung. Die Kernaufgabe der quantitativen Marktforschung ist die möglichst exakte Beschreibung der Marktlage und -entwicklung. Im Zentrum stehen Zahlen und Daten, wie z. B. Marktanteile, Marktvolumen, Wettbewerberverhalten,
Angebots- und Nachfrageverhalten. Dazu werden umfangreiche Stichproben mit relativ hohen Datensätzen benötigt. Die Auswertung der Daten erfolgt EDV-basiert mit Hilfe spezieller Statistik-Programme wie beispielsweise SPSS (Hofte-Frankhauser/Wälty 2009: 11).

Das Hauptanliegen der qualitativen Marktforschung ist das Verstehen, d. h. die Erklärung des Abnehmerverhaltens. Im Zentrum steht die Analyse von Wirkungszusammenhängen. Die Hauptthemen bei der qualitativen Marktforschung sind eher komplexe, nicht quantifizierbare »softe Faktoren«, beispielweise Erkenntnisfaktoren wie Werte, Motive, Einstellungen, Handlungsbeweggründe etc. Dazu werden in den meisten Fällen kleinere Stichproben und spezielle Erhebungs- und Auswertungstechniken (z. B. persönliche Interviews) eingesetzt (Hofte-Frankhauser/Wälty 2009: 11).

Die Datenerhebung erfolgt mittels Sekundär- oder Primärmarktforschung. Gegenstand der Sekundärmarktforschung (Engl. »Desk Research «) ist die Beschaffung von Daten, die bereits zu einem früheren Zeitpunkt und für andere Zwecke erhoben wurden. Dieses Datenmaterial wird im Hinblick auf das vorliegende Marktforschungsproblem aufbereitet und analysiert. Bei der Sekundärmarktforschung bedient man sich sowohl innerbetrieblicher als auch außerbetrieblicher Informationsquellen. $\mathrm{Zu}$ den innerbetrieblichen Quellen zählen u. a. Kunden-Datenbanken und Verkaufs- oder Reklamationsstatistiken. Bei den außerbetrieblichen Informationsquellen handelt es sich beispielsweise um Publikationen von Institutionen, Verbänden, Fachverlagen, Marktforschungsinstituten oder Forschungsergebnisse von Hochschulen. Sekundärdaten sind tendenziell relativ kostengünstig und schnell verfügbar. Jedoch

\begin{tabular}{|c|c|c|c|}
\hline \multirow[b]{2}{*}{ Charakterisierung } & \multicolumn{3}{|l|}{ Erhebungstechniken } \\
\hline & Befragung & Beobachtung & Test \\
\hline Definition & $\begin{array}{l}\text { Datenerhebungsmeth., bei der eine } \\
\text { Person mit gezielten Fragen zur An- } \\
\text { gabe der gewünschten Informationen } \\
\text { bewegt werden soll. }\end{array}$ & $\begin{array}{l}\text { Zielgerichtete und planmäßige } \\
\text { Erfassung sinnlich wahrnehmbarer } \\
\text { Sachverhalte zum Zeitpunkt ihres } \\
\text { Geschehens durch beobachtende } \\
\text { Personen oder Instrumente. }\end{array}$ & $\begin{array}{l}\text { Wiederholbare, unter kontrollierten } \\
\text { Bedingungen ablaufende Versuchs- } \\
\text { anordnung, in der vermutete kausale } \\
\text { Zusammenhänge zweier oder mehre- } \\
\text { rer Faktoren durch Veränderung der } \\
\text { Testgröße überprüft werden. }\end{array}$ \\
\hline Arten & $\begin{array}{l}\text { Quantitative Befragung } \\
\text { Qualitative Befragung }\end{array}$ & $\begin{array}{l}\text { Feldbeobachtung } \\
\text { Laborbeobachtung }\end{array}$ & $\begin{array}{l}\text { Labortest } \\
\text { Markttest }\end{array}$ \\
\hline Beispiele & $\begin{array}{l}\text { Erhebung der Umsatzbewegungen } \\
\text { im Detailhandel } \\
\text { Geschmacksvergleich zwischen } \\
\text { eigenem neuem Schoggimüesli und } \\
\text { Konkurrenzprodukt }\end{array}$ & $\begin{array}{l}\text { Kundenlaufstudie im Detailhandel } \\
\text { Beobachtung des Einkaufsverhaltens } \\
\text { Handhabungsbeobachtungen }\end{array}$ & $\begin{array}{l}\text { Einfluss verschiedener Produk- } \\
\text { designs auf Kaufbereitschaft der } \\
\text { Abnehmer } \\
\text { Auswirkung unterschiedlicher Regal- } \\
\text { platzierungen auf Absatz }\end{array}$ \\
\hline
\end{tabular}

Abb. 1: Informationsquellen der Primärmarktforschung (Maranghino-Singer 2005: 16) 
sind sie oftmals zu wenig spezifisch für die konkrete Fragestellung und/oder nicht aktuell genug (Böhler 2004: 63). Die Primärmarktforschung (Engl. »Field Research «) kommt dann zum Einsatz, wenn für die Lösung des Marktforschungsproblems neue Daten beschafft und aufbereitet werden müssen (Hofte-Frankhauser/Wälty 2009: 54). Die folgende Abbildung liefert eine Übersicht über relevante Informationsquellen der Primärmarktforschung und deren Erhebungstechniken.

Im Zusammenhang mit Fragetechniken ist eine Unterscheidung in offene und geschlossene Fragen üblich. Offene Fragen liefern i.d.R. komplexere Hintergrundinformationen, verlangen aber vom Befragten, dass er seine Antworten selbst formuliert. Bei geschlossenen Fragen werden die Antwortkategorien vorgegeben und können bspw. durch ein »Kreuz« oder »Haken« durch den Befragten gekennzeichnet werden (Böhler 2004: 99). Geschlossene Fragestellungen haben den Vorteil einer quantifizierbaren Auswertung.

Für eine Marktforschungsstudie ist $u$. a. die nachfolgende Schrittfolge empfehlenswert (Herrmann et al. 2008: 8):

1. Problemformulierung und Festlegung des Untersuchungsdesigns

2. Bestimmung der Verantwortlichkeiten

3. Festlegung der Datenerhebungsmethode

4. Stichprobenauswahl

5. Gestaltung des Erhebungsinstrumentes

6. Durchführung der Datenerhebung

7. Editieren und Kodierung der Daten

8. Datenanalyse und -interpretation

9. Präsentation der Ergebnisse

Die Studie zu marketingrelevanten Informationen bezüglich der Präferenzen von Kunden beim Kauf eines neuen Fernsehgerätes wurde in Anlehnung an obige Schrittfolge strukturiert.

\section{Methodik}

Im Mittelpunkt der Studie steht die Fragestellung, ausschlaggebenden Faktoren für oder gegen den Kaufentscheid eines neuen Fernsehgerätes im Zuge einer Primärerhebung herauszufiltern (1. Schritt: Problemformulierung und Festlegung des Untersuchungsdesigns). Das Projekt wurde von Studierenden im 2. Semester des Masterstudienganges »Business Management «, des Fachbereichs Betriebswirtschaft/Wirtschaftsinformatik unter Anleitung ihres betreuenden Professors ent- wickelt und durchgeführt (2. Schritt: Bestimmung der Verantwortlichkeiten).

Der Fragebogen stellt ein Instrument der Datenerhebung dar (3. Schritt: Festlegung der Datenerhebungsmethode), welches regelmäßig in den Wirtschafts- und Sozialwissenschaften sowie in der Psychologie Verwendung findet (SDI Research 2010). Der Aufbau eines Fragebogens sowie die Gestaltung und Fragestellung spielen eine wesentliche Rolle in Bezug auf die erfolgreiche Umsetzung eines Marktforschungsprojekts. Anstelle eines mit unzureichender Vorsicht konzipierten Fragebogens, sollte dieser mit Bedacht und ausreichend Zeit entwickelt werden, um der gewünschten Qualität gerecht zu werden. Gleichermaßen ist es empfehlenswert, den konstruierten Fragebogen einem Vor-Test zu unterziehen (LIS Bremen 2006).

Unter Berücksichtigung dieser Herangehensweise, ergab sich für die Studierenden zunächst die Notwendigkeit einer gründlichen Recherche zu den Themen Markenbildung und Fernsehgerätemarkt. Die Studierenden beschäftigten sich mit entsprechender Literatur und bereits erprobten Fragebögen, um möglichst viele Anregungen zu erhalten, mit deren Hilfe es möglich war, einen individuell passenden Fragebogen zur erfolgreichen Umsetzung des Projektziels zu entwickeln.

Bei der Generierung der Fragestellung waren alle Gruppenmitglieder beim Brainstorming (IBIM 2010) beteiligt, um zu diskutieren, welche der Fragestellungen besonders dringlich sind. Im Ergebnis haben die Studierenden der Marktforschungsgruppe einen gemeinsam entwickelten Fragebogen in einem Feldversuch an 10 Probanden im Dezember 2009 getestet (Vor-Test) und im Nachgang modifiziert. Im Ergebnis, d. h. in der modifizierten Endfassung des Fragebogens, wurden sowohl offene als auch geschlossene Fragen verwendet. Unter anderem wurden auch anonymisierte, soziodemographische Fragestellungen, wie beispielsweise das Geschlecht formuliert, um entsprechende Hintergrundinformationen für ein Kundensegmentiertes Marketing zu gewinnen (Broda 2006). Die Hauptstudie erfolgte im Zeitraum zwischen Januar und Juni 2010. Für die Stichprobe wurden Mitarbeiterinnen und Mitarbeiter der Technischen Hochschule Wildau [FH], sowie potentielle Interviewpartner aus dem Kreis der Familien und Bekannten der Studierenden herangezogen. Regional ist die Befragung damit auf den Raum BerlinBrandenburg konzentriert (4. Schritt: Stichprobenauswahl). Insgesamt wurden 420 standardisierte Fragebögen erstellt, 390 Fragebögen auf elektronischem Wege 
versandt und 30 Fragebögen im Rahmen von persönlichen Interviews verwendet. Insgesamt sind 71 Fragebögen beantwortet worden von denen 69 verwertbare Fragebögen für die Auswertung herangezogen werden konnten. Das entspricht einer Rücklaufquote von 16 Prozent. (5. Schritt: Gestaltung des Erhebungsinstrumentes und 6. Schritt: Durchführung der Datenerhebung). Für die Projektumsetzung wurde der Raum 320 im neu geschaffenen Marktforschungslabor im Haus 100 an der Technischen Hochschule Wildau [FH] genutzt. Die Studierenden haben die Antworten aus den Fragebögen übernommen, kodiert und mithilfe des Statistikprogramms SPSS ausgewertet. Insgesamt haben 35 männliche und 30 weibliche Interviewpartner an der Befragung teilgenommen. 4 Befragte wollten zu ihrem Geschlecht keine Aussage machen (7.Schritt: Editieren und Kodierung der Daten). Die Datenanalyse und -interpretation lieferte Ergebnisse, die wie folgt dargestellt werden können (gemäß Schritte 8 und 9):

\section{Datenauswertung}

\section{Kaufpreis}

Der Großteil (rund 51 Prozent) der befragten Teilnehmerinnen und Teilnehmer der Stichprobe, avisiert einen Kaufpreis zwischen $€ 800$ und $€ 1.200$ für ein neues Fernsehgerät. Dieser Bereich könnte daher als Massenmarkt interpretiert werden. 20 Prozent sind bereit, bis $€ 600$ für ein neues Fernsehgerät auszugeben. Immerhin sind 16 Prozent der Befragten offen, $€ 1.500$ und mehr! für ein neues Gerät zu investieren. Ebenfalls interessant ist, dass rund 9 Prozent gar keine Präferenz bezüglich des Kaufpreises haben. Diese Gruppe bildet aus Unternehmenssicht ein besonders interessantes Potenzial für Marketingspezifische Instrumente (emotionale Ansprache durch eine Marke).

Die Ergebnisse der Erhebung haben keine wirklich signifikanten, geschlechterspezifischen Unterschiede, in Bezug auf den Kaufpreis, ermittelt. Tendenziell haben die weiblichen Befragten eine Präferenz im unteren Preissegment (bis € 400), während die männlichen Befragten in der Kategorie bis $€ 1.000$ überwiegen. Um in dieser Kategorie verlässlichere Daten zu erhalten, müsste die Stichprobe in einer Folgeerhebung wesentlich erweitert werden.

\begin{tabular}{|l|r|r|r|r|}
\hline Gültig & Häufigkeit & Prozent & $\begin{array}{r}\text { Gültige } \\
\text { Prozente }\end{array}$ & $\begin{array}{r}\text { Kumulierte } \\
\text { Prozente }\end{array}$ \\
\hline keine Präferenz & 6 & 8,7 & 8,7 & 8,7 \\
\hline bis 200 Euro & 4 & 5,8 & 5,8 & 14,5 \\
\hline bis 400 Euro & 4 & 5,8 & 5,8 & 20,3 \\
\hline bis 600 Euro & 6 & 8,7 & 8,7 & 29,0 \\
\hline bis 800 Euro & 13 & 18,8 & 18,8 & 47,8 \\
\hline bis 1000 Euro & 12 & 17,4 & 17,4 & 65,2 \\
\hline bis 1200 Euro & 10 & 14,5 & 14,5 & 79,7 \\
\hline bis 1500 Euro & 3 & 4,3 & 4,3 & 84,1 \\
\hline bis 2.000 Euro & 5 & 7,2 & 7,2 & 91,3 \\
\hline über 2.000 Euro & 3 & 4,3 & 4,3 & 95,7 \\
\hline keine Angabe & 3 & 4,3 & 4,3 & 100,0 \\
\hline Gesamt & 69 & 100,0 & 100,0 & \\
\hline
\end{tabular}

Abb. 2: Marktsegmentierung nach Fernsehgerätekaufpreis

\section{Technologie}

Nur rund die Hälfte der Befragten wissen um den Unterschied von »HD-ready« und »full HD«. Dabei ist zu beachten, dass die Bildqualität von modernen LCD und Plasmageräten ohne full HD $(1.920 \times 1.080$ Pixeln $)$ herkömmlichen Röhrengeräten noch immer unterlegen ist. Hinzu kommt, dass Ausstellungs- und Vorführgeräte im Handel, oftmals mit einem Blu-ray Rekorder (Signalgeber) gekoppelt sind, der technologisch momentan die höchste Bildauflösung (nämlich full HD) garantiert. Das heißt, 43 Prozent der Befragten (Antwortkategorien: ist mir egal bzw. nein) ist theoretisch für eine irreführende Werbung anfällig. Diese potenti-

\begin{tabular}{|c|c|c|c|c|c|c|c|c|c|c|c|c|}
\hline Anzahl & $\begin{array}{l}\text { maximaler } \\
\text { keine }\end{array}$ & $\begin{array}{l}\text { Kaufpre } \\
\text { bis }\end{array}$ & bis & bis & bis & bis & bis & bis & bis & über & keine & Gesamt \\
\hline 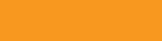 & Präferenz & 200 & 400 & 600 & 800 & 1.000 & 1.200 & & & & & . \\
\hline weiblich & 3 & 3 & 3 & 3 & 5 & 3 & 5 & 1 & 2 & 2 & 0 & 30 \\
\hline männlich & 3 & 1 & 1 & 3 & 7 & 9 & 5 & 2 & 3 & 1 & 0 & 35 \\
\hline keine Angabe & 0 & 0 & 0 & 0 & 1 & 0 & 0 & 0 & 0 & 0 & 3 & 4 \\
\hline Gesamt & 6 & 4 & 4 & 6 & 13 & 12 & 10 & 3 & 5 & 3 & 3 & 69 \\
\hline
\end{tabular}

Abb. 3: männlich vs weiblich * maximaler Kaufpreis Kreuztabelle Mehrheit haben 
ellen Kunden sehen bspw. ein Vorführgerät im Handel (z. B. mit full HD und an ein blu-ray Gerät gekoppelt), wissen aber nicht um die unterschiedliche Bildschirmauflösung und machen ihren Kaufentscheid in erster Linie vom Preis (bzw. irreführenden Werbeversprechen) abhängig. Wenn das neue Fernsehgerät (HDready und analoges Signal) dann zu Hause benutzt wird, ist die Wahrscheinlichkeit einer Enttäuschung gegeben. Natürlich bleiben auch full-HD Geräte ohne digitales Signal weit unter ihren Möglichkeiten (Bildschirmauflösung).

\begin{tabular}{|l|r|r|r|r|}
\hline Gültig & $\begin{array}{l}\text { Häufig- } \\
\text { keit }\end{array}$ & Prozent & $\begin{array}{c}\text { Gültige } \\
\text { Prozente }\end{array}$ & $\begin{array}{l}\text { Kumulierte } \\
\text { Prozente }\end{array}$ \\
\hline ist mir egal & 7 & 10,1 & 10,1 & 10,1 \\
\hline Ja & 36 & 52,2 & 52,2 & 62,3 \\
\hline Nein & 23 & 33,3 & 33,3 & 95,7 \\
\hline keine Angabe & 3 & 4,3 & 4,3 & 100,0 \\
\hline Gesamt & 69 & 100,0 & 100,0 & \\
\hline
\end{tabular}

Abb. 4: Unterschied HD_ready_vs_fullHD

Da in den kommenden Jahren nach Vorstellungen der Industrie das sog. digitale (Bezahl-) Fernsehen (ausgerichtet auf full HD Geräte), zunehmend den Markt bestimmen wird, ist ein relativ früher Folgekauf (nämlich der momentanen Besitzer von HD-ready Geräten), zur Freude der den Markt beherrschenden Fernsehgerätehersteller aus Asien, wie bspw. Samsung und Sony vorprogrammiert. Die Marken Samsung (23 Prozent) und Sony (22 Prozent) wurden mehrheitlich von den Befragten als präferierten Marken genannt (z. B. wurde Loewe überhaupt nicht genannt). Philips gehört nach den Ergebnissen der Studie ebenfalls zu den bevorzugten Marken in Bezug auf den Kauf eines Fernsehgerätes (13 Prozent). In diesem Zusammenhang muss man aber wissen, dass Philips mittlerweile den überwiegenden Teil seiner Wertschöpfungsaktivitäten nach Asien, insbesondere China, verlagert hat.
In Übereinstimmung mit der momentanen Marktentwicklung verifizieren auch die Ergebnisse der Studie eine klare Präferenz für LCD-Geräte. Rund 65 Prozent würden sich für ein LCD-Gerät entscheiden. Dabei gibt es bei den männlichen Befragten eine relativ höhere Präferenz für LCD-Geräte (80 Prozent), als bei den weiblichen Befragten (53 Prozent). Die weiblichen Befragten sind tendenziell aufgeschlossener für ein Plasmagerät (30 Prozent) im Vergleich zu den männlichen Interviewpartnern (14 Prozent).

\begin{tabular}{|l|r|r|r|r|r|r|}
\hline $\begin{array}{l}\text { Anzahl } \\
\text { männlich } \\
\text { vs weiblich }\end{array}$ & andere & Röhren & LCD & Plasma & $\begin{array}{l}\text { keine } \\
\text { Angabe }\end{array}$ & Gesamt \\
\hline weiblich & 2 & 2 & 16 & 9 & 1 & 30 \\
\hline männlich & 1 & 1 & 28 & 5 & 0 & 35 \\
\hline keine & 0 & 0 & 1 & 0 & 3 & 4 \\
\hline Angabe & & & & & & 4 \\
\hline Gesamt & 3 & 3 & 45 & 14 & 4 & 69 \\
\hline
\end{tabular}

Abb. 5: männlich vs weiblich * Bilddarstellung Kreuztabelle

Mehr als die Hälfte der Befragten (58 Prozent) hat zu dem Thema Zusatzfunktionen (z. B. Ambilight von Philips, DVBT-Tuner) eine eher ablehnende Haltung (völlig unwichtig, unwichtig oder relativ unwichtig) bzw. hat keine Meinung oder wollte keine Angabe gemachen. Tendenziell haben aber die männlichen Befragten eine höhere Affinität für Zusatzfunktionen als die weiblichen Befragten.

\section{Informationsbeschaffung und Kaufort}

Der Großteil der Befragten informiert sich vor einem Kaufentscheid anhand von Testberichten (30 Prozent). Der Anteil der Befragten die sich vor einem Kauf überhaupt nicht informieren ist auf der anderen Seite ebenfalls relativ hoch (28 Prozent). Während die männlichen Befragten tendenziell auf Testberichte als Informationsgrundlage zurückgreifen, verlassen sich die

\begin{tabular}{|c|c|c|c|c|c|c|c|c|c|}
\hline $\begin{array}{l}\text { Anzahl } \\
\text { männlich vs } \\
\text { weiblich }\end{array}$ & $\begin{array}{l}\text { Wichtigkeit } \\
\text { völlig } \\
\text { unwichtig }\end{array}$ & $\begin{array}{l}\text { Zusatzfunkti } \\
\text { unwichtig }\end{array}$ & $\begin{array}{l}\text { nen } \\
\text { relativ } \\
\text { unwichtig }\end{array}$ & $\begin{array}{l}\text { keine } \\
\text { Meinung }\end{array}$ & $\begin{array}{l}\text { relativ } \\
\text { wichtig }\end{array}$ & wichtig & $\begin{array}{l}\text { extrem } \\
\text { wichtig }\end{array}$ & $\begin{array}{l}\text { keine } \\
\text { Angabe }\end{array}$ & Gesamt \\
\hline weiblich & 6 & 3 & 2 & 10 & 5 & 3 & 1 & 0 & 30 \\
\hline männlich & 3 & 3 & 2 & 7 & 7 & 8 & 5 & 0 & 35 \\
\hline keine Angabe & 0 & 0 & 0 & 1 & 0 & 0 & 0 & 3 & 4 \\
\hline Gesamt & 9 & 6 & 4 & 18 & 12 & 11 & 6 & 3 & 69 \\
\hline
\end{tabular}

Abb. 6: männlich vs weiblich * Wichtigkeit Zusatzfunktionen Kreuztabelle 
weiblichen Befragten eher auf Empfehlungen aus dem Familien- und Freundeskreis.

Der überwiegende Teil der Interviewpartner kauft ein neues Fernsehgerät in großen Elektronikkaufhäusern (56 Prozent). Darüber hinaus wird anhand des ausgewerteten Datenmaterials deutlich, dass die weiblichen Befragten - im Vergleich zu den männlichen Teilnehmern der Stichprobe - einem Kauf im Internet wesentlich kritischer gegenüberstehen.

\section{Schlussbemerkung}

Die vorliegende Studie zu marketingrelevanten Informationen in Zusammenhang mit dem Kauf eines neuen Fernsehgerätes wurde im Wesentlichen von einer Arbeitsgruppe, bestehend aus acht Studierenden des Masterstudienganges Business Management im 2. Semester initiiert, konzipiert und durchgeführt. Die Marktforschungsstudie ist ein Beispiel für die angewandte Forschung und praxisnahe Lehre, unter weitestgehender Einbeziehung der Studierenden, an der Technischen Hochschule Wildau [FH]. Erkenntnisse aus der Auswertung von 69 Fragebögen, könnten in einer späteren Projektphase, mit einem wesentlich erweiterten Stichprobenumfang, vor dem Hintergrund einer möglichst hohen Repräsentativität, verifiziert werden. Die Projektgruppe der Studierenden nebst Professor bedankt sich bei allen Mitarbeiterinnen und Mitarbeitern der Technischen Hochschule Wildau (FH), die sich trotz intensiver Arbeitsbelastung, die Zeit genommen haben, den Fragebogen auszufüllen und damit ihren ganz persönlichen Beitrag für eine interessante, praxisnahe Lehre im Sinne unserer Studierenden, geleistet haben.

\section{Literaturverzeichnis}

Adjouri, N. 2002: Die Marke als Botschafter - Markenidentität bestimmen und entwickeln. Wiesbaden: Gabler Verlag.

Böhler, H. 2004: Marktforschung. Stuttgart: Kohlhammer Verlag. Broda, S. 2006: Marktforschungs-Praxis: Konzepte, Methoden, Erfahrungen. Wiesbaden: Gabler Verlag.

Esch, F., Tomczak,T., Kernstock, J. und Langner, T. 2006: Corporate Brand Management. 2. Aufl., Wiesbaden: Gabler Verlag. Herrmann, A., Homburg, C. und Klarmann, M. 2008: Handbuch Marktforschung: Methoden - Anwendungen - Praxisbeispiele. Wiesbaden: Gabler Verlag.

Hofte-Frankhauser, K., Wälty, H. 2009: Marktforschung: Grundlagen mit zahlreichen Beispielen, Repetitionsfragen mit Lösungen und Glossar. Zürich: Compendio Verlag.

Hohn, S. 2008: Public Management - Marketing Management für den öffentlichen Sektor. 2. Aufl., Wiesbaden: Gabler Verlag.

IBIM 2010: http://www.ibim.de/techniken/3-1.htm (Zugriff am 18. Juni 2010).

Kotler, P., Bliemel, F. 2001: Marketing-Management - Analyse, Planung und Verwirklichung. 10. Aufl., Stuttgart: Schäffer-Poeschel.

Kuß, A., Eisend, M. 2010: Marktforschung: Grundlagen der Datenerhebung und Datenanalyse. Wiesbaden: Gabler Verlag.

LG 2010: http://www.lg.com/de/index.jsp (Zugriff am 18. Juni 2010).

LIS Bremen 2010: http://www.lis.bremen.de/sixcms/media.php/13/ Skript\%20Fragebogenerstellung.pdf (Zugriff am 28. Juni 2010).

\begin{tabular}{|c|c|c|c|c|c|c|c|}
\hline $\begin{array}{l}\text { Anzahl } \\
\text { männlich vs } \\
\text { weiblich }\end{array}$ & $\begin{array}{l}\text { Inforn } \\
\text { nein }\end{array}$ & $\begin{array}{l}\text { tonsquellen vor } \\
\text { ja = Freunde } \\
\text { und Familie }\end{array}$ & $\begin{array}{l}\text { m Kauf } \\
\text { ja = Testbericht }\end{array}$ & $\mathrm{ja}=$ Internet & $\begin{array}{l}\text { ja = Verkäufe- } \\
\text { rin/Verkäufer }\end{array}$ & keine Angabe & Gesamt \\
\hline weiblich & 8 & 10 & 8 & 2 & 1 & 1 & 30 \\
\hline männlich & 11 & 6 & 13 & 4 & 0 & 1 & 35 \\
\hline keine Angabe & 0 & 0 & 0 & 0 & 0 & 4 & 4 \\
\hline Gesamt & 19 & 16 & 21 & 6 & 1 & 6 & 69 \\
\hline
\end{tabular}

Abb. 7: männlich vs weiblich * Informationsquellen vor dem Kauf Kreuztabelle

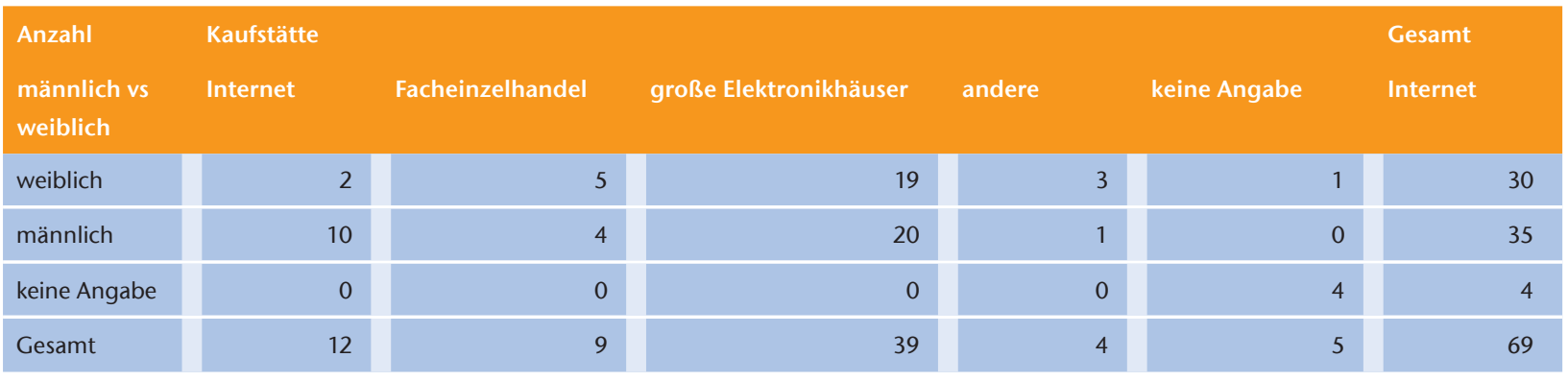

Abb. 8: männlich vs. weiblich * Kaufstätte Kreuztabelle 
Maranghino-Singer, B., »ISB. «2005: www.isb.uzh.ch/studium/courses05/pdf/0281_marketing.pdf (Zugriff am 2. Mai 2010).

Meffert, H., Burmann, C. und Koers, M., »Ephorie.de.« 2001: http:// www.ephorie.de/pdfs/Meffert-Markenmanagement.pdf (Zugriff am 7. Juni 2010).

SDI Research 2010: http://www.sdi-research.at/lexikon/befragung. html (Zugriff am 29. Juni 2010).

Zednik, A., Strebinger, A. 2005: Marken-Modelle der Praxis: Darstellung - Analyse und kritische Würdigung. Wiesbaden: Universitätsverlag.

\section{Autoren}

\section{Sascha Bartels}

Technische Hochschule Wildau [FH]

\section{Fabian Bräuner}

Technische Hochschule Wildau [FH]

\section{Prof. Dr. Mario Glowik}

Fachbereich Betriebswirtschaft/Wirtschaftsinformatik

Technische Hochschule Wildau [FH]

mario.glowik@th-wildau.de

\section{Roman Hawlitzky}

Technische Hochschule Wildau [FH]

\section{Claudia Kottke}

Technische Hochschule Wildau [FH]

\section{Claudia Liedtke}

Technische Hochschule Wildau [FH]

\section{Julia Mücke}

Technische Hochschule Wildau [FH]

\section{Matthias Schidzick}

Technische Hochschule Wildau [FH]

Christopher Weiß

Technische Hochschule Wildau [FH] 